\title{
Avances preliminares en la determinación de los balances de agua en parcelas forestales y pastizales mediante la aplicación del modelo hidrológico de Témez
}

\author{
Virt, Laura E1.; Currie, Héctor M. \\ Hidrología Agrícola, Facultad de Ciencias Agrarias, UNNE - Sgto. Cabral 2131 - Corrientes, Argentina Tel (0379) 4427589 \\ (int. 129) lauravirt@gmail.com ${ }^{1}$ Becaria Secretaria General de Ciencia y Técnica-UNNE
}

\begin{abstract}
Resumen
Con el objetivo de establecer el balance hídrico en parcelas con pastizales y forestales y evaluar potenciales diferencias entre las mismas se determinaron las componentes de dicho balance en base a una planilla de Cálculo Excel ${ }^{\circledR}$, mediante la aplicación del Modelo Hidrológico de Témez, en el cual las componentes halladas fueron: ETP (evapotranspiración), Po (umbral de escorrentía), $\delta$ (déficit máximo de humedad del suelo), Excedente de agua, ETR (evapotranspiración real), Hi (almacenamiento de agua en el suelo), Infiltración, Escurrimiento, Almacenamiento Subterráneo, Aporte de agua subterráneo y Aporte Total. Estas variables fueron analizadas estadísticamente mediante la Prueba de T para muestras apareadas, la cual permite probar la hipótesis de igualdad de medias, cuando se toman observaciones de a pares desde las dos distribuciones que se comparan (pastizal y forestal), teniendo una muestra de tamaño $\mathrm{n}$ de pares de observaciones, cada miembro de un par proveniente de una distribución. El balance en parcelas forestales arrojó un valor de $(110,35 \mathrm{~mm})$ que resultó menor, comparado con el balance en los pastizales $(135,4 \mathrm{~mm})$, esto es debido en gran parte al componente intercepción del dosel y, al tener solo un dato de balance de cada caso en particular, se procedió a aplicar el análisis estadístico a sus componentes, para verificar si existen o no diferencias significativas entre las mismas. Al realizar dicho análisis estadístico se concluyó que, en cuanto a las precipitaciones y escurrimiento, no existen diferencias significativas entre las mismas, si bien cabe aclarar que en el caso del lote forestal a esas precipitaciones se les descontó el agua interceptada por el dosel, por lo tanto el valor absoluto disminuye en parte.
\end{abstract}

Palabras Clave: Balance Hidrológico, Agua Disponible, Intercepción, Almacenamiento Subterráneo, Evapotranspiración.

\begin{abstract}
Summary
With the objective to determinate the water balance in plots of grassland and other with forestry plantation and assess potential differences between the samples, the components of this balance were determined using Excel program, applying the Témez Hydrologic Model, and the following components were found: ETP (evapo-transpiration), Po (run-off threshold), $\delta$ (humidity maximum soil deficit), water surplus, RET (real evapo-transpiration), Hi (water soil stock), infiltration, run-off, sub terrain stock, sub terrain water supply and total supply. These parameters were statistically analyzed using the $\mathrm{T}$ Test for matched samples, which allows to prove the hypothesis of equality of averages, when pair observations are taken from both distributions under comparison (grassland and forestry land), considering a sample of " $n$ " observations pairs, each member of a pair coming from a single distribution. The balance in the forestry samples had a value of $110,35 \mathrm{~mm}$ that was lower releted to the balance in the grassland $(135,4 \mathrm{~mm})$ due to the important rain interception of the canopy., The fact of have one measure of the balance for each case the statistic analysis of their components was applied, to verify significant differences between them. After the statistic analysis the conclusion in relation to the rains and run-off was there is no significant difference between them, although in the forestry sample the raining rate has a discount due to the canopy interception and therefore the absolute value were lower.
\end{abstract}

Key Words: Hydrologic Balance, Water Available, Intercept, Underground storage, evapotranspiration.

\section{INTRODUCCIÓN}

El desarrollo de la forestación en la Argentina ha ido sustituyendo tierras de uso ganadero y/o agrícola por plantaciones de Eucalyptus y Pinus, causando esto preocupación respecto a sus efectos sobre los recursos naturales, particularmente aguas y suelos. Este nuevo uso de la tierra involucra fuertes cambios en el funcionamiento del ecosistema. Las forestaciones plantean nuevas oportunidades productivas, pero también compromisos con servicios esenciales 
que los ecosistemas brindan, tales como agua dulce, o la regulación hidrológica de cuencas. En este tipo de ecosistemas, una fracción del agua de la precipitación es interceptada por la copa de los árboles, evaporándose directamente parte de ella sin llegar al suelo. El agua que llega al piso forestal, infiltra saturando (o no) el suelo, o escurre en forma superficial, alimentando a los cursos de agua. Las raíces toman el agua que infiltra en el suelo y la trasladan hacia las hojas, donde es utilizada en el proceso de fotosíntesis, siendo luego transpirada. La suma de la fracción de agua transpirada y la evaporada directamente desde la planta conforman la evapotranspiración. El agua que no es absorbida por las plantas, percola hacia estratos más profundos. La proporción de cada uno de estos flujos respecto a la precipitación bruta depende de diferentes factores, tales como la estructura del bosque, las características del follaje, del tronco y de la corteza, las características del sitio, la pendiente del terreno, el manejo silvicultural, y las actividades de aprovechamiento como así también de las características climáticas y edáficas. Los ecosistemas forestales interceptan una fracción importante del agua de lluvia, que así no llega al suelo y cuentan con un sistema radicular capaz de absorber mayor cantidad de agua que otros cultivos. Esto implica impactos de la forestación sobre algunas propiedades de los suelos, diferentes a los que podrían esperarse en regiones naturalmente boscosas o selváticas que son reforestadas. Las forestaciones establecidas afectan al recurso agua al liberar como vapor hacia la atmósfera parte del agua que normalmente alcanzaría a los ríos y al acuífero. El conocimiento del destino de las precipitaciones y del patrón de recarga de los acuíferos es decisivo para entender el efecto que una plantación forestal ejerce sobre el recurso hídrico y su manejo. La mayor capacidad relativa de uso del agua por parte de las plantaciones forestales de eucaliptus y pinos, lo convierte en un freno a la recarga de los acuíferos. Esta característica ha hecho de la forestación el eje de discusiones acerca de ventajas y desventajas en áreas con excesos de agua o limitaciones hídricas (Rebori et al., 1997).

Se puede citar el trabajo de Díaz (2006), a partir del cual se concluyó que, como toda actividad humana que hace uso del suelo y de los recursos naturales, la actividad forestal produce impactos negativos y positivos sobre el ambiente. La información indica que la tendencia y magnitud de los cambios producidos por las forestaciones son variables y dependen de diversos factores. Es por ello conveniente disponer de información generada localmente.

Según Silveira y otros (2006), mediante líneas de tendencia para coeficiente de escorrentía anual y estacional, confeccionaron un esquema en la que expresan la tendencia a una reducción porcentual de los escurrimientos, producto del desarrollo forestal en la cuenca.

El fuerte consumo de agua por los bosques es largamente conocido, a tal punto que en el pasado se ha planteado a la forestación como una herramienta de regulación hidrológica de cuencas. Tras el establecimiento de plantaciones forestales en la cabecera de cuencas se esperaba lograr una disminución de los picos de máximo caudal tras lluvias grandes, reduciendo el peligro de inundaciones y una redistribución de estos potenciales excesos en el tiempo, capaz de generar caudales más constantes durante el año (Jobbágy et al., 2008).

\section{El Balance de Agua}

El balance de agua del suelo resulta de computar las ganancias y pérdidas del sistema hidrológico.

El cálculo del balance de agua del monte forestal se basa en:

$$
P=I \pm S \pm Q \pm E T
$$

P es la lluvia medida a campo abierto.

I es la pérdida de lluvia por intercepción del dosel.

$\mathrm{S}$ es el cambio en el contenido del agua del suelo o agua disponible.

Q es la percolación (-) o el ascenso capilar (+) hacia o desde capas más profundas o almacenamiento subterráneo.

ET es la pérdida de agua por evaporación del suelo y transpiración del monte.

\section{OBJETIVO}

El objetivo del presente trabajo fue determinar mediante la aplicación del Modelo Hidrológico de Témez (Témez, 1977) y a través de una planilla de cálculo, tanto el balance de agua en parcelas con pastizales, como con forestales, los componentes de dicho balance y evaluar potenciales diferencias entre los mismos.

\section{MATERIALES Y MÉTODOS}

Los materiales utilizados fueron: un registro de datos de precipitaciones (1930-2007) del Establecimiento Rincón de Luna, ubicado en la Ciudad de Concepción, Corrientes (latitud: $28^{\circ} 46^{\prime} 67^{\prime \prime}$ y longitud: $\left.58^{\circ} 23^{\prime} 33^{\prime \prime}\right)$, el cual dista del sitio de estudio unos $20 \mathrm{~km}$, aproximadamente, otro registro (mayo 2010-abril 2011) proveniente de la lluvia interceptada por los bidones colocados en el lote con forestales de 9 años de edad, análisis de suelos e infiltraciones realizados in-situ, tanto en el lote con pastizales, como en el forestal.

Para medir la intercepción de los forestales frente a las precipitaciones se colocaron perfiles de goma (Figura $\mathrm{N}^{\circ} 1$ ) en forma de $\mathrm{U}-$ estos perfiles son neu- 
máticos usados, cortados de manera que se genere una sección en U- , colocados alrededor del fuste en forma circular. Estos descargan en un tanque recolector inclinado $5^{\circ}$, para facilitar el escurrimiento a lo largo del tronco y se ubican a una altura de DAP (diámetro a la altura del pecho) sobre la superficie del suelo, a los efectos de evitar el salpicado desde la superficie del suelo.

El tanque recolector en el que descargaron los perfiles tiene una capacidad de 20 litros. Cada tanque está provisto de un tubo externo transparente, conectado al fondo y una escala graduada (en mm). Los dispositivos recolectores se colocaron en grupos de cuatro, sobre fustes de árboles representativos de los existentes en la plantación, en posiciones alejadas de los bordes del lote; el criterio para la elección del árbol está dada por el DAP.

Para determinar el balance de estas parcelas se utilizó el Método de Témez, el cual, a partir de variables de entrada, concretamente precipitación y evapotranspiración potencial y los parámetros hidrológicos, permite obtener los distintos almacenamientos, humedad en el suelo y volumen del acuífero y de las variables de salida del ciclo hidrológico, evapotranspiración y escorrentía total, obtenida esta última como suma de la escorrentía superficial y subterránea (Figura $\mathrm{N}^{\circ} 2$ ).

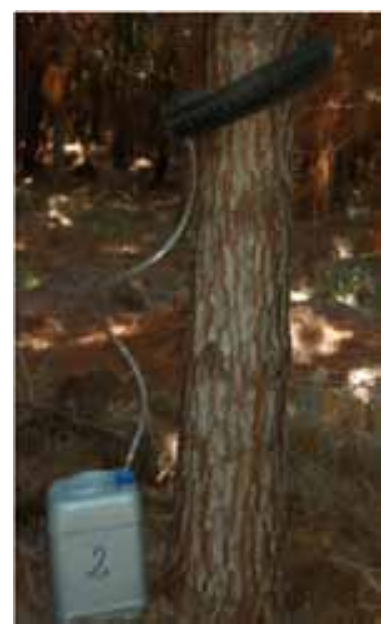

Figura $N^{\circ}$ 1: Perfil de goma alrededor de un árbol y tanque recolector de agua de precipitaciones.

Figura $\mathbf{N}^{\circ}$ 2: descripción esquemática del flujo de agua del Modelo de Témez (extraído de Témez, 1997).
Las entradas al modelo son la precipitación y la evapotranspiración potencial, obtenida a partir de registros locales de temperaturas mensuales en la estación meteorológica del Establecimiento Rincón de Luna.

Los parámetros del modelo son: capacidad máxima de almacenamiento de humedad en el suelo, coeficiente de excedente -que regula el denominado umbral de escorrentía-, capacidad máxima de infiltración y coeficiente de recensión de los acuíferos.

\section{Fase Superficial}

El modelo Témez, plantea una serie de ecuaciones conceptuales para el cálculo de los flujos y almacenamiento de agua en las celdas en las que se ha discretizado el terreno.

El cálculo del excedente de agua es, básicamente, función de la precipitación $\left(\mathrm{P}_{\mathrm{i}}\right)$, del déficit de humedad en el suelo $\left(\mathrm{H}_{\text {máx }}-\mathrm{H}_{\mathrm{i}}-1\right)$ y de la evapotranspiración potencial $\left(\mathrm{EP}_{\mathrm{i}}\right)$, y tiene como expresión (Témez, 1977):

$$
\begin{gathered}
P_{i} \leq P_{0} T_{i}=0 \\
P_{i}>P_{0} T_{i}=\left(P_{i}-P_{0}\right)^{2} / P_{i}+\delta-2 P_{0}
\end{gathered}
$$

Donde:

$$
\begin{gathered}
\delta=H_{\max }-H_{i}-1+E P_{i} \\
P_{0}=C\left(H_{\max }-H_{i}\right)
\end{gathered}
$$

Siendo en cada celda:

$\mathrm{P}_{\mathrm{i}}$ : precipitación en el mes $\mathrm{i}(\mathrm{mm})$

$\mathrm{T}_{\mathrm{i}}$ : excedente de agua en el mes $\mathrm{i}(\mathrm{mm})$

$\mathrm{H}_{\max }$ : capacidad máxima de almacenamiento de agua en el suelo (mm)

$\mathrm{H}_{\mathrm{i}}$ : almacenamiento de agua en el suelo en el mes i-1 (mm)

$\mathrm{EP}_{\mathrm{i}}$ : evapotranspiración potencial en el mes i (mm)

$\mathrm{C}$ : parámetro de excedente, que toma valores del orden de 0,3 .

Una vez definidas las condiciones iniciales, con la asistencia de una planilla de cálculo se pueden resolver las distintas ecuaciones del modelo Témez, comenzando por el parámetro de excedente $\mathrm{T}_{i}$, cuya formulación se ha descrito anteriormente (ecuaciones 1 a 4), y por el cálculo de la evapotranspiración potencial $\mathrm{EP}_{\mathrm{i}}$, necesaria para la estimación posterior de la ecuaciones 5 y 6 en cada celda.

Otras variables necesarias para la resolución del modelo en su fase superficial son el almacenamiento de agua en el suelo $\left(\mathrm{H}_{\mathrm{i}}\right)$ y la evapotranspiración real $\left(E_{i}\right)$ en cada celda en el mes $i$, y que se obtienen mediante las siguientes expresiones: 


$$
\begin{gathered}
H_{i}=\operatorname{máx}\left[0,\left(H_{i-1}+P_{i}-T_{i}-E P_{i}\right)\right] \\
E_{i}=\min \left[\left(H_{i-1}+P_{i}-T_{i}\right), E P_{i}\right]
\end{gathered}
$$

\section{Fase Subterránea}

La infiltración al acuífero por lluvia directa $\left(\mathrm{I}_{\mathrm{i}}\right)$ en cada celda es función del excedente de agua $\left(\mathrm{T}_{\mathrm{i}}\right)$ y del parámetro de infiltración máxima $\left(\mathrm{I}_{\text {máx }}\right)$ y adopta la siguiente expresión (Témez, 1977):

$$
I_{i}=I_{\max } \cdot T_{i} /\left(T_{i}+I_{\text {max }}\right)
$$

El modelo de Témez (1977) asume que la recarga al acuífero en cada celda coincide con la infiltración. Una vez calculada la recarga en cada una de las celdas pertenecientes a los distintos acuíferos, el modelo realiza su integración en los recintos que los definen y aplica el conocido modelo unicelular de forma agregada. La evolución del volumen almacenado en el acuífero y su descarga a la red de drenaje superficial o al mar se realiza mediante las siguientes expresiones:

$$
\begin{array}{r}
V_{i}=V_{i-1} \cdot e^{-\alpha . \Delta t}+R_{i} / \alpha(1-e-\alpha \cdot \Delta t) \\
A_{\text {subi }}=V_{i-1}-V_{i}+R_{i} \text { (9) }
\end{array}
$$

siendo:

$\alpha$ : coeficiente de agotamiento del acuífero (meses-1)

$\Delta \mathrm{t}$ : intervalo temporal (mes)

$\mathrm{R}_{\mathrm{i}}$ : recarga al acuífero en el mes i ( $\left.\mathrm{mm} / \mathrm{mes}\right)$

$V_{i}$ : volumen almacenado en el acuífero en el mes i (mm)

$\mathrm{A}_{\text {subi }}$ : aportación subterránea correspondiente al

\begin{tabular}{|c|c|c|c|c|c|c|c|c|c|c|c|c|c|}
\hline & MIIS (mm.mes s. $\left.^{-1} \mathbf{b a}^{-1}\right)$ & PP (mm.mes $\left.{ }^{-4} \cdot h a^{-4}\right)$ & $\begin{array}{c}\text { ETP } \\
\text { (mme-mes- } \\
\text { 1.ha-1) }\end{array}$ & $\begin{array}{c}\text { Po } \\
\text { (mm.mes' } \\
\text { 'ha') }\end{array}$ & $\begin{array}{c}6 \\
\text { (mames } \\
\left.{ }^{1} \cdot \mathrm{ha}^{-1}\right)\end{array}$ & $\begin{array}{l}\text { EXCED. } \\
\underset{(m m . m e s}{ } \\
\left.{ }^{1} \cdot \text { ha }^{-3}\right)\end{array}$ & $\begin{array}{c}\text { ETR } \\
\text { (mm.mes } \\
{ }^{1} \text {.mat) }\end{array}$ & $\underset{\substack{\text { (mm.mes' } \\
\text { 'ba') }}}{\mathbf{H i}}$ & $\begin{array}{c}\text { NF } \\
\text { (mmanes' } \\
\text { "hat) }\end{array}$ & $\begin{array}{c}\text { ESC } \\
\text { (mm.mes' } \\
{ }^{1} \text {.ha."-3) }\end{array}$ & $\begin{array}{c}\text { ALM. } \\
\text { SCBT } \\
\text { (mm.mes' } \\
\left.{ }^{1} \cdot \text { a a }^{4}\right)\end{array}$ & $\begin{array}{c}\text { APORT. } \\
\text { SUBT } \\
\text { (mm.mes' } \\
\left.{ }^{1} \text { ha' }^{4}\right)\end{array}$ & $\begin{array}{l}\text { APORT. } \\
\text { TOTAL } \\
\text { (mmimes' } \\
\text { 'that) }\end{array}$ \\
\hline \multirow{13}{*}{ 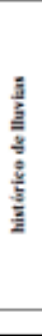 } & 1 & 124.054 & 77 & 13.85 & 1231 & 55,3 & 68.7 & 0 & 48,4 & 69 & 15,1 & 33.3 & 40.2 \\
\hline & 2 & 139 & 366 & 13.85 & 1328 & 04,2 & 74.8 & 0 & 55 & 9,1 & 18,7 & 515 & 60.6 \\
\hline & 3 & 143,915 & 124,8 & 13.85 & 171 & 589 & 85 & 0 & 51,1 & 78 & 17,8 & 52 & 59,3 \\
\hline & 4 & 199,426 & 190,5 & 13.85 & 236.6 & 57,5 & 101.9 & 0 & 50,1 & 7,5 & 17,4 & 50.5 & 579 \\
\hline & 5 & 79.8298 & 245,8 & 13.85 & 295 & 12,5 & 673 & 0 & 12,1 & 0,4 & 5.5 & 24 & 24,4 \\
\hline & 6 & 629574 & 299.3 & 13.85 & 345.4 & 6,3 & 56.6 & 0 & 6.2 & 0,1 & 2.5 & 9.2 & 9.3 \\
\hline & 7 & 51.6393 & 322,2 & 13.85 & 368.4 & 3.6 & 48 & 0 & 3.6 & 0 & 1.4 & 47 & 48 \\
\hline & 8 & 44.8723 & 278,5 & 15,85 & 324.7 & 2,8 & 42,1 & 0 & 28 & 0 & 1 & 3,2 & 3,2 \\
\hline & 9 & 74,0638 & 243,6 & 13.85 & 294.8 & 10,6 & 63,4 & 0 & 10,3 & 0,3 & 3,3 & 3 & 8.3 \\
\hline & 10 & 123,915 & 165,6 & 13.85 & 211.7 & 39,3 & 34.6 & 0 & 35,7 & 3,6 & 11.3 & 27.5 & 31.2 \\
\hline & 11 & 122319 & 114,2 & 13.85 & 100.4 & 46,1 & 76.2 & 0 & 41.2 & 49 & 14 & 38.7 & 43.6 \\
\hline & 12 & 115,106 & 75.1 & 13.85 & 1213 & 49.1 & 66 & 0 & 43.6 & 5.5 & 15 & 426 & 48.1 \\
\hline & promedio & 103,4 & 185,9 & 13.83 & 232,1 & 33,9 & 69.6 & 0 & 30 & 39 & 10.3 & 28.8 & 32.6 \\
\hline
\end{tabular}
mes i (mm/mes).

Cuadro $\mathbf{N}^{\circ}$ 1: Resultados de la planilla de cálculo para la parcela forestal

\begin{tabular}{|l|c|}
\hline Unidad de Suelo & Serie Chavarria \\
\hline Agua disponible en el Perfil & 19,65 \\
\hline Hmax (A. disp.X 0,916) & 18 \\
\hline Coeficiente de ajuste Cpo & 0,3 \\
\hline ETP media mensual & 75 \\
\hline Parámetro de infiltración (Imáx) & 386 \\
\hline Parámetro de descarga (@- alfa) & 2,325 \\
\hline
\end{tabular}

La aportación total es igual a la parte de excedente $\left(\mathrm{T}_{\mathrm{i}}\right)$ no infiltrada $\left(\mathrm{I}_{\mathrm{i}}\right)$, más la aportación subterránea $\left(\mathrm{A}_{\text {subi }}\right)$ :

$$
A_{\text {toti }}=\left(T_{i}-I_{i}\right)+A_{\text {subi }}=A_{\text {supi }}+A_{\text {subi }}
$$

siendo:

$\mathrm{A}_{\text {supi }}$ aportación superficial del mes $\mathrm{i}\left(\mathrm{mm} \mathrm{mes}^{-1}\right)$

$\mathrm{A}_{\text {toti }}$ aportación total durante el mes i ( $\left.\mathrm{mm} \mathrm{mes}^{-1}\right)$.

Para analizar los componentes del balance de cada caso en particular se utilizó la "Prueba de T-Student" para muestras apareadas, la cual permite probar la hipótesis de igualdad de medias cuando se toman observaciones de a pares desde las dos distribuciones que se comparan, teniendo una muestras de tamaño $n$ de pares de observaciones, cada miembro de un par proveniente de una distribución. Para realizar esta prueba se utilizó el producto InfoStat ${ }^{\circledR}$, que requiere un archivo con dos columnas: una para las observaciones provenientes de la distribución 1 y otra para las de la distribución 2. Si el valor obtenido al aplicar este estadístico nos diera $p<0,0001$, sugiere el rechazo de la hipótesis $\mathrm{H}_{0}: \mu \mathrm{d}=0$, es decir existen diferencias estadísticamente significativas entre las dos distribuciones.

\section{Resultados}

Los resultados de los balances fueron:

Balance Pastizal: $\mathrm{P}=\mathrm{AD}$ (agua disponible) + $\mathrm{Q}$ (almacenamiento subterráneo) $+\mathrm{ET}$

\section{$50,4 \mathrm{~mm}+10,3 \mathrm{~mm}+75 \mathrm{~mm}=135,40 \mathrm{~mm}$}

Balance Forestal: $\mathrm{P}=\mathrm{AD}$ (agua disponible) + $\mathrm{Q}$ (almacenamiento subterráneo) + ET

\section{$19,65 \mathrm{~mm}+15,7 \mathrm{~mm}+75 \mathrm{~mm}=110,35 \mathrm{~mm}$}

En los cuadros $\mathrm{N}^{\circ} 1$ y 2 , se observan los datos de entrada de la planilla de cálculo: agua disponible $(\mathrm{mm})$, calculada a partir de valores obtenidos de los muestreos de suelo, ETP (evapotranspiración, mm mes $^{-1}$ ), extraídas del libro Caracterización Agroclimática de la Provincia de Corrientes, del Consejo 
Cuadro $N^{\circ}$ 2: Resultados de la planilla de cálculo para la parcela pastizal.

\begin{tabular}{|c|c|c|c|c|c|c|c|c|c|c|c|c|c|}
\hline \multicolumn{3}{|c|}{ Unidad de Suelo } & \multicolumn{3}{|c|}{ Serie Chavarria } & & & & & & & & \\
\hline \multicolumn{3}{|c|}{ Agua disponible en el Perfil } & \multicolumn{3}{|c|}{50,4} & & & & & & & & \\
\hline \multicolumn{3}{|c|}{$\mathrm{Hmax}$ (A. disp. X 0,916) } & \multicolumn{3}{|c|}{46,2} & & & & & & & & \\
\hline \multicolumn{3}{|c|}{ Coeficiente de ajuste Cpo } & \multicolumn{3}{|c|}{0,3} & & & & & & & & \\
\hline \multicolumn{3}{|c|}{ ETP media mensual } & \multicolumn{3}{|c|}{75} & & & & & & & & \\
\hline \multicolumn{3}{|c|}{ Parámetro de infiltración (Imáx) } & \multicolumn{3}{|c|}{386} & & & & & & & & \\
\hline \multicolumn{3}{|c|}{ Parámetro de descarga (@-alfa) } & \multicolumn{3}{|c|}{2,325} & & & & & & & & \\
\hline & IIES (mm.mes . $\left.^{-1} \mathbf{a}^{-1}\right)$ & PP (mm.mes $\left.\cdot h a^{-1}\right)$ & $\begin{array}{c}\text { ETP } \\
\text { (mmames- } \\
\text { 1.ha-1) }\end{array}$ & $\begin{array}{c}\text { Po } \\
\text { (mm.mes } \\
\text { '.ha'b }\end{array}$ & $\begin{array}{c}b \\
\text { (mmmes } \\
{ }^{3} \cdot \mathrm{ha}^{-2} \text { ) }\end{array}$ & $\begin{array}{l}\text { EXCED. } \\
\text { (mm.mes } \\
\left.{ }^{3} \cdot \mathrm{ha}^{-3}\right)\end{array}$ & $\begin{array}{c}\text { ETR } \\
\text { (mm.mes } \\
\left.{ }^{1} \cdot \mathbf{m a}^{4}\right)\end{array}$ & $\begin{array}{c}\text { Hi } \\
\text { (mm.mes } \\
{ }^{2} \mathbf{h a}^{2} \text { ) }\end{array}$ & $\begin{array}{c}\text { NF. } \\
\text { (mmmes } \\
\text { '.ha') }\end{array}$ & $\begin{array}{c}\text { ESC. } \\
\text { (mm.mes } \\
\left.{ }^{3} \cdot \text { ha }^{-3}\right)\end{array}$ & 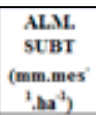 & $\begin{array}{l}\text { APORT. } \\
\text { SUBT } \\
\text { (mm.mes' } \\
\text { 'ma') }\end{array}$ & $\begin{array}{l}\text { APORT. } \\
\text { TOTAI } \\
\text { (mam.mes' } \\
\text { '.ha') }\end{array}$ \\
\hline \multirow{13}{*}{ 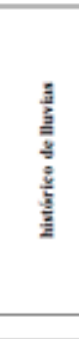 } & 1 & 118,4 & 27.8 & 5.4 & $\$ 5,7$ & 83,3 & 27.8 & 7,4 & 63.5 & 14,8 & 21,4 & 47.1 & 61.3 \\
\hline & 2 & 133.2 & 353 & 3,19 & 459 & 979 & 353 & 75 & 78,1 & 19.8 & 26.5 & 73 & 923 \\
\hline & 3 & 132.3 & 45.8 & 3,16 & 56,3 & 91,5 & 45,8 & 25 & 74 & 17,5 & 25,7 & 74.8 & 923 \\
\hline & 4 & 153,7 & 703 & 4,65 & 36 & 98,4 & 59.8 & 0 & $n+2$ & 19,3 & 26.6 & 762 & 95,5 \\
\hline & 5 & $\infty 6.1$ & 93.8 & 5.4 & 111,7 & 22.1 & 44 & 0 & 20.9 & 1.2 & 9.1 & 38,4 & 39.6 \\
\hline & 6 & 62,4 & 129 & 5.4 & 147 & 16,4 & 46 & 0 & 15,7 & 0,7 & 5.8 & 19 & 19.7 \\
\hline & 7 & 36.5 & 141 & 5,4 & 199 & 5,2 & 31.3 & 0 & 52 & 0,1 & 2,2 & 88 & 89 \\
\hline & 8 & 44.6 & 117 & 5.4 & 135 & 9.1 & 353 & 0 & 39 & 0.2 & 3 & 8.1 & 83 \\
\hline & 9 & 60,4 & 102,8 & 5.4 & 120.7 & 17.8 & 42.6 & 0 & 17 & 0,8 & 5.6 & 14,4 & 15.2 \\
\hline & 10 & 123,2 & $\infty$ & 5,4 & 34 & 70,7 & 525 & 0 & 99,7 & 109 & 19,1 & 46.1 & 57 \\
\hline & 11 & 121,1 & 43.5 & 5,4 & 61.5 & 779 & 43.2 & 0 & 6,8 & 13,1 & 22.2 & 619 & 75 \\
\hline & 12 & 106.3 & 27 & 5.4 & 45 & 72.5 & 27 & 6.8 & 61 & 11.5 & 21.2 & 619 & 73,4 \\
\hline & promedio & 96.5 & 74.9 & 5 & 91,5 & 55.1 & 40.9 & 2 & 45,9 & 9.1 & 15.7 & 44,1 & 53,3 \\
\hline
\end{tabular}

Cuadro $\mathbf{N}^{\circ}$ 3: Resultados del estadístico, Prueba de T para muestras apareadas.

\begin{tabular}{|c|c|c|c|c|c|c|c|c|}
\hline \multicolumn{9}{|c|}{ Prueba T (muestras apareadas) } \\
\hline \multicolumn{9}{|c|}{ Almacenamiento } \\
\hline Obs 1 & Obs. 2 & $\mathbf{N}$ & Media (dif) & Media 1 & Media 2 & DE (dif) & $\mathbf{T}$ & Bilateral \\
\hline alm SUBT Forestal & alm SUBT Pastizal & 12 & 5,44 & 15,71 & 10,27 & 2,88 & 6,55 & $<0,0001$ \\
\hline \multicolumn{9}{|c|}{ Aporte } \\
\hline Obs 1 & Obs. 2 & $\mathbf{N}$ & Media (dif) & Media 1 & Media 2 & DE (dif) & $\mathbf{T}$ & Bilateral \\
\hline aporte SUBT Forestal & aporte SUBT Pastizal & 12 & 15,38 & 44,14 & 28,77 & 7,62 & 6,99 & $<0,0001$ \\
\hline \multicolumn{9}{|c|}{ Precipitaciones } \\
\hline Obs 1 & Obs. 2 & $\mathbf{N}$ & Media (dif) & Media 1 & Media 2 & DE (dif) & $\mathbf{T}$ & Bilateral \\
\hline PP Forestal & PP Pastizal & 12 & $-6,91$ & 96,52 & 103,43 & 5,6 & $-4,27$ & 0,0013 \\
\hline \multicolumn{9}{|c|}{ Escurrimiento } \\
\hline Obs 1 & Obs. 2 & $\mathbf{N}$ & Media (dif) & Media 1 & Media 2 & DE (dif) & $\mathbf{T}$ & Bilateral \\
\hline esc. Forestal & esc. Pastizal & 12 & 5,32 & 9,16 & 3,84 & 4,56 & 4,04 & 0,002 \\
\hline
\end{tabular}

Federal de Inversiones (Castro, 1991). En cuanto a los datos de precipitación $(\mathrm{mm})$, se realizó un promedio mensual histórico utilizando el registro de precipitaciones del Establecimiento Rincón de Luna.

La planilla de cálculo arrojó: ETP (evapotranspiración), Po (umbral de escorrentía), $\delta$ (déficit máximo de humedad del suelo), Excedente de agua, ETR (evapotranspiración real), Hi (almacenamiento de agua en el suelo), Infiltración, Escurrimiento, Almacenamiento Subterráneo, Aporte de agua subterráneo y Aporte Total, todos estos datos en mm mes ${ }^{1} \mathrm{ha}^{-1}$.

\section{DISCUSIÓN}

En cuanto al escurrimiento calculado por el Modelo de Témez, si bien no incide directamente como componente en el cálculo del Balance, teniendo en cuenta el trabajo de Silveira y colaboradores (2006), en el cual se afirma que las plantaciones forestales tienen una tendencia a disminuir porcentualmente los escurrimientos. En este caso, desde un punto de vista estadístico, no existen diferencias significativas entre los valores de escurrimiento del pastizal y del pinar.

Rebori (1997), demuestra que las plantaciones forestales exóticas tienen mayor capacidad relativa de uso del agua. En el presente trabajo ello se ve reflejado al analizar la componente "agua disponible", la cual, en valores absolutos, es mucho menor en el pinar (19,65 mm) que en el pastizal (50,4 mm).

Al tener solo un dato de balance de cada caso en particular, se procedió a aplicar un análisis estadístico a sus componentes, para verificar si existen o no diferencias significativas entre las mismas. Los resultados de este análisis pueden observarse en el cuadro $\mathrm{N}^{\circ} 3$.

\section{CONCLUSIONES}

Es posible concluir que mediante el Modelo Hidrológico de Témez es factible determinar, tanto los balances hídricos de los dos ecosistemas en cuestión, como sus componentes. 
La diferencia entre los valores de los balances de estos ecosistemas, $110,35 \mathrm{~mm}$ para la parcela forestal y $135,4 \mathrm{~mm}$ para la parcela pastizal, es debido en parte a la componente "intercepción por el dosel".

Luego de realizar el análisis estadístico se concluye que, en lo que se refiere a precipitaciones y escurrimiento, no existen diferencias significativas entre las mismas, si bien cabe aclarar que en el caso del lote forestal, a esas precipitaciones se les descontó el agua interceptada por el dosel, por lo que el valor absoluto disminuyó en parte.

\section{BIBLIOGRAFÍA}

Castro, G. (1991), Caracterización Agroclimática de la Provincia de Corrientes, Consejo Federal de Inversiones, Tomo II. 237 páginas.

Díaz, D. (2006), XXI ${ }^{\mathrm{a}}$ Jornadas Forestales de Entre Ríos Argentina Forestal, Investigadora del INTA E.E.A Concordia.
Jobbágy, E.; Nosetto, M.; Santoni, C.; Balde, G. (2008), "El desafío eco hidrológico de las transiciones entre sistemas leñosos y herbáceos en la llanura Chaco-Pampeana", vol.18, no.3. Grupo de Estudios Ambientales, IMASL - Universidad Nacional de San Luis y CONICET, San Luis, Argentina.

Rebori, G.; Díaz, R. (1997), "Redistribución de las lluvias y balance de agua de una plantación de Eucalyptus dan en el sur de Santa Fe" (primera parte), Centro Nacional de Investigaciones Agropecuarias, INTA Castelar.

Silveira, L.; Alonso, J.; Martínez, L. (2006), “Efecto de las plantaciones forestales sobre el recurso agua en el Uruguay", Agrociencia. Vol. X N 2 páginas $75-93$.

Software estadístico InfoStat ${ }^{\circledR}$, versión 2010.

Témez, J. (1977), Modelo matemático de transformación Precipitación- Aportación, ASINEL 1977. 